\title{
Rapid Processing of a Global Feature in the ON Visual Pathways of Behaving Monkeys
}

\author{
Jun Huang 1,2, Yan Yang ${ }^{3}$, Ke Zhou 4,5,, Xudong Zhao ${ }^{3}$, Quan Zhou ${ }^{3}$, Hong Zhu ${ }^{2,7,8}$, \\ Yingshan Yang ${ }^{9}$, Chunming Zhang ${ }^{10}$, Yifeng Zhou ${ }^{1,3 *}$ and Wu Zhou ${ }^{2,7,8 *}$ \\ ${ }^{1}$ CAS Key Laboratory of Brain Function and Disease, and School of Life Sciences, University of Science and Technology of \\ China, Hefei, China, ${ }^{2}$ Department of Otolaryngology and Communicative Sciences, University of Mississippi Medical Center, \\ Jackson, MS, United States, ${ }^{3}$ State Key Laboratory of Brain and Cognitive Science, Institute of Biophysics, Chinese \\ Academy of Sciences, Beijing, China, ${ }^{4}$ College of Psychology and Sociology, Shenzhen University, Shenzhen, China, ${ }^{5}$ Center \\ for Language and Brain, Shenzhen Institute of Neuroscience, Shenzhen, China, ${ }^{6}$ Shenzhen Key Laboratory of Affective and \\ Social Cognitive Science, Shenzhen University, Shenzhen, China, ${ }^{7}$ Department of Neurobiology and Anatomical Sciences, \\ University of Mississippi Medical Center, Jackson, MS, United States, ${ }^{8}$ Department of Neurology, University of Mississippi \\ Medical Center, Jackson, MS, United States, ${ }^{9}$ Primate Research Center of Jin Gang International, Haikou, China, \\ ${ }^{10}$ Department of Otolaryngology, First Affiliated Hospital, Shanxi Medical University, Taiyuan, China
}

OPEN ACCESS

Edited by:

Thomas Serre,

Brown University, United States

Reviewed by:

Chou P. Hung,

United States Army Research

Laboratory, United States

Juha Silvanto,

University of Westminster,

United Kingdom

*Correspondence:

Yifeng Zhou

zhouy@ustc.edu.cn

Wu Zhou

wzhou@umc.edu

Specialty section:

This article was submitted to

Perception Science,

a section of the journal

Frontiers in Neuroscience

Received: 20 April 2017 Accepted: 10 August 2017

Published: 25 August 2017

Citation:

Huang J, Yang $Y$, Zhou $K$, Zhao $X$, Zhou $Q$, Zhu $H$, Yang Y, Zhang $C$, Zhou Y and Zhou W (2017) Rapid

Processing of a Global Feature in the

ON Visual Pathways of Behaving

Monkeys. Front. Neurosci. 11:474.

doi: 10.3389/fnins.2017.00474
Visual objects are recognized by their features. Whereas, some features are based on simple components (i.e., local features, such as orientation of line segments), some features are based on the whole object (i.e., global features, such as an object having a hole in it). Over the past five decades, behavioral, physiological, anatomical, and computational studies have established a general model of vision, which starts from extracting local features in the lower visual pathways followed by a feature integration process that extracts global features in the higher visual pathways. This local-to-global model is successful in providing a unified account for a vast sets of perception experiments, but it fails to account for a set of experiments showing human visual systems' superior sensitivity to global features. Understanding the neural mechanisms underlying the "global-first" process will offer critical insights into new models of vision. The goal of the present study was to establish a non-human primate model of rapid processing of global features for elucidating the neural mechanisms underlying differential processing of global and local features. Monkeys were trained to make a saccade to a target in the black background, which was different from the distractors (white circle) in color (e.g., red circle target), local features (e.g., white square target), a global feature (e.g., white ring with a hole target) or their combinations (e.g., red square target). Contrary to the predictions of the prevailing local-to-global model, we found that (1) detecting a distinction or a change in the global feature was faster than detecting a distinction or a change in color or local features; (2) detecting a distinction in color was facilitated by a distinction in the global feature, but not in the local features; and (3) detecting the hole was interfered by the local features of the hole (e.g., white ring with a squared hole). These results suggest that monkey $\mathrm{ON}$ visual systems have a subsystem that is more sensitive to distinctions in the global feature than local features. They also provide the behavioral constraints for identifying the underlying neural substrates.

Keywords: topological perception, visual search, feature integration, saccade, behaving monkeys 


\section{INTRODUCTION}

Extensive investigations over the past five decades have led to a general model of visual information processing, which starts from extracting local features of the retinal images in the lower visual pathways followed by integrating the local features to extract global features in the higher visual pathways (Hubel, 1988). Consistent with this model, neurons in the lateral geniculate nucleus (LGN) exhibit fine center-surround receptive fields that support extracting edges in the retinal images (Hubel, 1988; Van Hooser et al., 2013); neurons in the primary visual cortex (V1) exhibit elongated receptive fields that support extracting orientation of line segments (Hubel and Wiesel, 1962, 1963, 1977; Hubel, 1982; Tootell et al., 1998; Yacoub et al., 2008); neurons in the inferior temporal cortex, however, exhibit large receptive fields that support extracting more complex features of objects, such as hands and faces (Gross et al., 1972; Bruce et al., 1981; Bruce, 1982; Schwartz et al., 1983; Desimone, 1991). Behavioral studies provided further evidence to support the early feature detection and later feature integration theory. Using a visual search paradigm, Treisman and Gelade (1980) found that primitive features, such as color or orientation of line segments are extracted effortlessly, and in parallel over the entire visual field, and registered in special modules of feature maps. They suggested that in a later stage, focal attention is required to recombine the separate features for object recognition. Motivated by the anatomical, physiological and behavioral studies of visual systems, Marr (1982) proposed an influential computational system of vision, in which the simple components, and their geometric properties, typically as line segments with slopes, are used as representations of the retinal image in the early stages of its analysis. Over the past half century, the local-to-global theory of visual information processing has gained general acceptance and dominates the field of vision research.

Contrary to the early feature analysis approach, there is an early holistic registration approach, which suggests that perceptual processing is from global to local, i.e., wholes are coded prior to perceptual analysis of their separable properties or parts. Despite the general acceptance of the local-to-global approach, there is accumulating evidence for the global-to-local approach, including configural superiority (Weisstein and Harris, 1974; Navon, 1977), early detection of topological properties (Chen, 1982, 1990; Chen and Zhou, 1997), topological perception and long-range apparent motion (Chen, 1985; Zhuo et al., 2003; Wang et al., 2007; Zhou et al., 2010), detection of topological properties in bees (Chen et al., 2003), invariant shape processing in rats (see review, Zoccolan, 2015), visual perceptual learning (Ahissar and Hochstein, 2004), and holistic detection of Mooney faces, and objects (Bona et al., 2016). In particular, Chen (1982) and Chen et al. (2003) showed in humans and bees that two shapes (e.g., circle and ring) that were different in the hole feature (a global feature) were more discriminable than other pairs of shapes that were different in local features (e.g., circle and square). Nonetheless, the neural substrates underlying the global-to-local processes remain elusive, and there is a big knowledge gap in reconciling the local-to-global, and the global-to-local approaches. The main goal of the present study is to test the hypothesis that monkey visual systems rapidly detect the hole feature over local features. If successful, it not only adds support to the "global-first" theory, but also provides a robust platform for elucidating the neural mechanisms of processing global and local features by neurophysiological, and neuropharmacological approaches. This study was also motivated to reconcile the inconsistent reports in the limited monkey literature on processing the hole feature. While Komatasu and Ideura (1993) showed that a group of neurons in the monkey inferior temporal cortex were selectively activated by shapes with a hole, regardless of the shape of the holes, Shen et al. (2002) showed that hole precedence in monkeys was not in shape discrimination, but limited to face discrimination. In this study, we employed the pop-up visual search paradigm (Treisman and Gelade, 1980; Bichot and Schall, 1999) to directly compare the latencies to detect the targets that were different from the distractors in local features, global features, and color. Our results showed that monkey visual systems rapidly detected the hole feature, consistent with the human literature. Critical assessment of the local-to-global and global-to-local models will not only address the controversial but fundamental question of vision, i.e., what are the primitives of vision, but also provide important insights into cortical, and subcortical visual mechanisms in health and diseases.

\section{METHODS}

Two macaque monkeys (M1 and M2) were fitted to a head positioning platform. Eye movements were monitored by a magnetic search coil technique (Robinson, 1963; Judge et al., 1980; Zhou and King, 1998). Surgical procedures and experimental protocols were approved by the Institutional Animal Care and Use Committee at University of Mississippi Medical Center.

\section{Visual Stimuli and Eye Movement Measurement}

The visual stimuli were a set of geometric shapes $\left(2^{\circ}\right.$ in size $)$ arranged with equal distances $\left(4^{\circ}\right)$ to the central fixation point. In the set, one object was the target, the others were distractors, i.e., white circles. The target was different from the distractors in color (e.g., red circle), local features (e.g., white square), a global feature (e.g., white ring with a black hole) or their combinations (e.g., red ring). The visual stimuli were displayed on a SAMSUNG PN64F5300AF Plasma TV (PN64F5300) monitor driven by an NVIDIA GT 610 video card. The monitor was located at $140 \mathrm{~cm}$ in front of the monkey. It had a display area of $140 \times 80 \mathrm{~cm}$, with a resolution of $1,920 \times 1,080$ pixels. The refresh rate of the monitor was $60 \mathrm{~Hz}$. Using a Digital Lux meter LX1330B, the monitor background luminance was measured as $0.2 \mathrm{~cd} / \mathrm{m}^{2}$, the luminance of the white, red, green and blue surface was measured as $270,43,155$, and $76 \mathrm{~cd} / \mathrm{m}^{2}$, respectively. It is important to note that early topological perception studies in humans (Chen, 1982, 1990) presented black stimuli against a white background, therefore, demonstrating detection of topological properties in human OFF visual pathways. In this study, the stimuli were 


\section{A With target onset}

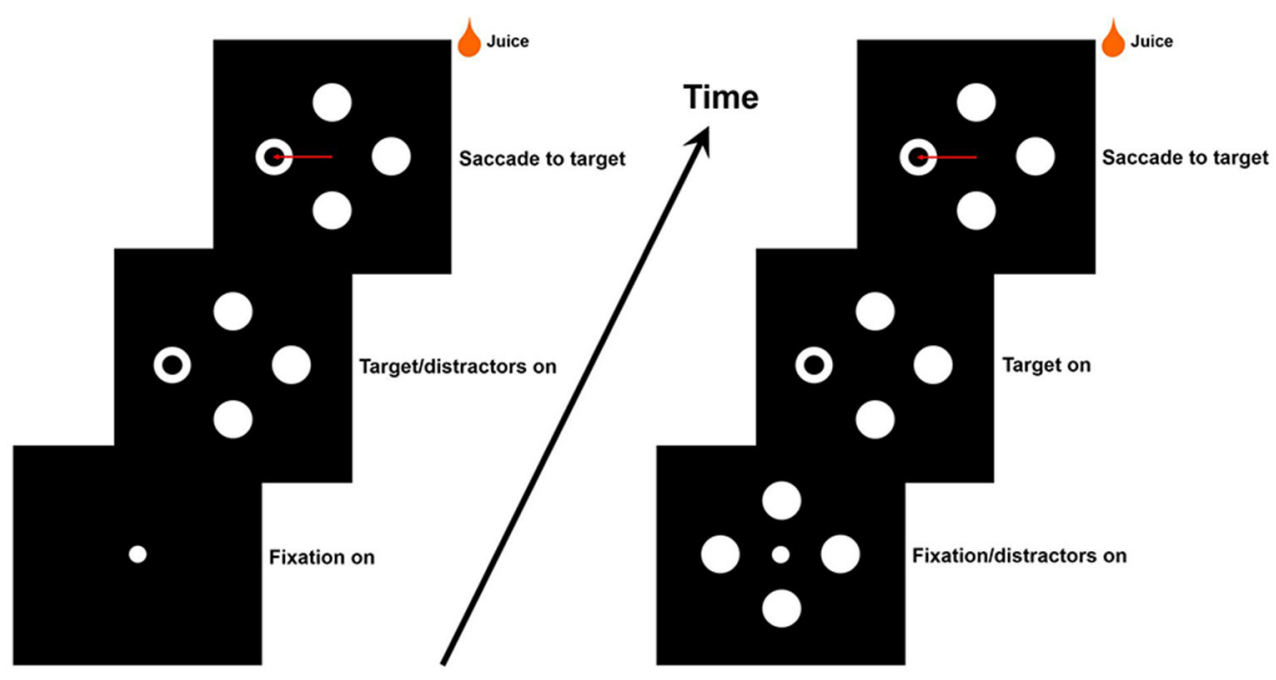

C

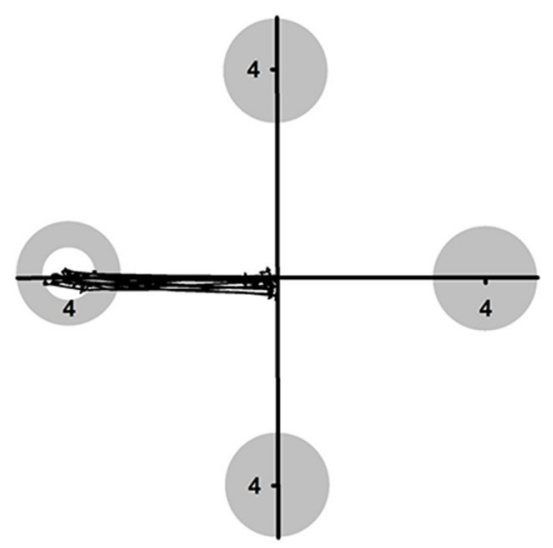

B Without target onset

FIGURE 1 | (A,B), Experimental paradigms in the with-onset and without-onset conditions target (right panel). Each black line represents one saccade trajectory.

presented against a black background and primarily activated monkey ON visual pathways. However, Yeh et al. (2009) showed that "black" responses dominate monkey V1 and later visual cortical areas. Thus, it is important to limit our conclusions to the $\mathrm{ON}$ visual pathways and further investigate how the monkey OFF visual pathways detect global features in future studies. Stimulus onset was determined by a photocell positioned in the upper left corner of the monitor. Monkeys viewed the stimuli binocularly and were trained to make saccades to fixate the target for juice rewards. Eye positions were calibrated by requiring the monkeys to fixate spots at known horizontal and vertical eccentricities.

\section{Experimental Conditions}

In the first experiment, the target and distractors appeared simultaneously after the fixation point $(500-700 \mathrm{~ms})$ was extinguished (Figure 1A). The target appeared randomly in one of the four or eight locations (four cardinal and four oblique directions) at eccentricities $4^{\circ}$. The target was different from distractors in color (red/green/yellow/blue circle vs. white circles), local features (white square vs. white circles), area (white ring target with different hole sizes or small white circle target with different diameters) or the global feature (white ring vs. white circles) (Figure 1C). In the second experiment, the distractors were presented first and after a fixation hold, one of the distractors was changed to the target while the central fixation point was turned off (Figure 1B). Unlike the first experiment, in which target onset was present in each trial, this experiment allowed us to selectively assess detecting a change in color, local features or the global feature. In the third experiment, the target was different from the distractors not only in color, but also in local features or the global feature. For example, the monkeys were asked to detect a red square from white circles, or a red ring from white circles. In the fourth experiment, the target was 
different from the distractors in both the global feature and local features. For example, the monkeys were asked to detect a white ring with a square-shape hole from white circles, or a white ring with a square-shape outline from white circles.

\section{Data Acquisition and Data Analysis}

The experiments were controlled by BESC (High Tech Serv, Rochester, NY), in which experimental conditions were formulated as temporal sequences of instructions to operate devices (turn on and off visual stimuli, check fixation, deliver a drop of juice, etc.). A CED Power 1401 system (Cambridge Electronics Devices, Cambridge, UK) was used for data acquisition. Signals of eye coils and photocells were sampled at $1 \mathrm{kHz}$ with 16-bit resolution. These signals were stored on a hard disk for offline analysis. Eye movement responses were analyzed using Spike2 (Cambridge Electronics Devices, Cambridge, UK), Matlab (Mathworks, Natick, MA) and SigmaPlot (Systat Software, San Jose, CA). Raw eye position data were filtered and differentiated with a band-pass of DC to $100 \mathrm{~Hz}$ to obtain eye velocity data. Trials in which monkeys made a saccade within $50 \mathrm{~ms}$ of the onset of the visual stimuli were rejected. Saccade onset was determined as the first point in the velocity trace over $20 \mathrm{deg} / \mathrm{s}$ (Figure 2A). The latency averages and percentage averages of correct trials were computed based on 250 trials in each condition (usually 5 sessions, 50 trials per session) (Figure 2B) (Bronstein and Kennard, 1987; Kalesnykas and Hallet, 1987; Smith and Van Gisbergen, 1989; Wenban-Smith and Findlay, 1991; Fischer and Weber, 1993; Fischer et al., 1993; Pare and Munoz, 1996; Zhou and King, 2002). ANOVA, paired $t$-test and post hoc comparisons were used to assess significance of the main effects and differences between conditions.

\section{RESULTS}

\section{Rapid Detection of the Ring Target}

Figure 3 shows that saccade latencies to the white ring target were shorter than that to the white square target, but similar to that to the red circle target for the two monkeys (M1 and M2). During the experiments, the target appeared randomly in one of the four or eight locations of the stimulus set. For clarity, our analysis was focused on the trials with the target at the two horizontal locations. Since set size (4 or 8 ) had no effect on saccade latencies (M1: $P>0.05 ; \mathrm{M} 2: P>0.05$ ), data analysis was focused on the results in set size 4 condition. ANOVA showed that the differences among saccade latencies to the three targets were significant $(P<0.001)$. Post-hoc comparisons further show that saccade latencies to the ring target were shorter than that to the square target (M1: $P<0.001 ; \mathrm{M} 2: P<0.001)$, but similar to that to the red circle target $(\mathrm{M} 1: P>0.05 ; \mathrm{M} 2: P$ $>0.05)$. For M1, detection of the ring target in the left visual field (LVF) had a latency of $155.01 \pm 0.86 \mathrm{~ms}$, which was not different from the latencies to detect the red circle target (154.39 $\pm 1.64 \mathrm{~ms}, P=0.744$ ), but was significantly shorter than the latencies to detect the square target $(187.13 \pm 1.58 \mathrm{~ms}, P<0.001)$ (Figure 3A, LVF). For M2, saccade latency to the ring target $(118.03 \pm 0.81 \mathrm{~ms})$ was similar to that to the red target $(124.87$ $\pm 1.32 \mathrm{~ms} ; P>0.05)$, but shorter than that to the square target $(142.11 \pm 2.32 \mathrm{~ms} ; P<0.001)$ (Figure 3B, LVF). Both monkeys correctly detected the targets in over $90 \%$ of the trials in LVF (M1: 93.7\%; M2: 92.9\%). Similar results were obtained for targets in the right visual field (RVF).

We also measured saccade latencies to targets with other colors, such as blue, green, and yellow. ANOVA showed that there was a significant main effect of color on saccade latency.
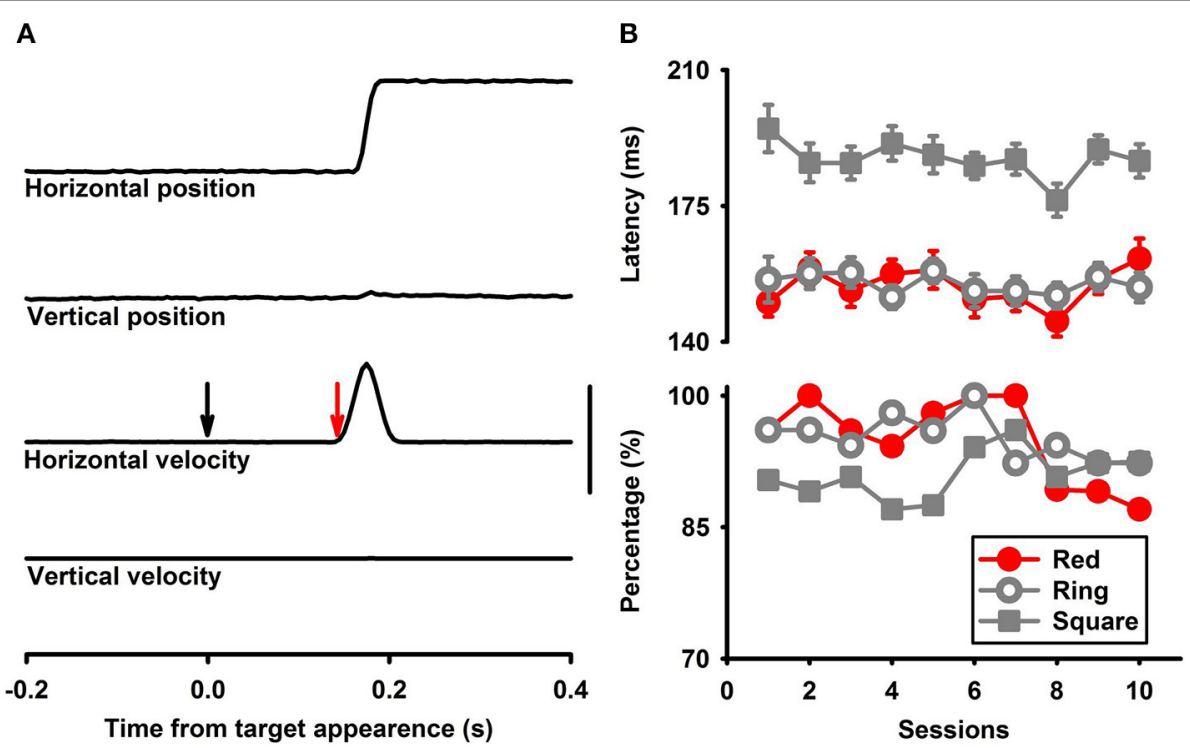

FIGURE 2 | (A) Horizontal and vertical eye position and velocity signals. The black arrow indicates target onset, and the red arrow indicates saccade onset. The vertical bar is $4^{\circ}$ for eye position and $150^{\circ}$ for eye velocity. (B) Representative saccade latencies (upper panel) and accurate rates (lower panel) in multiple sessions for $\mathrm{M} 1$. 


\section{A M1}
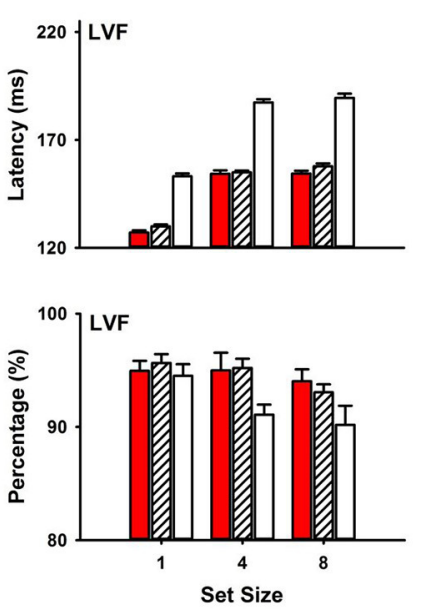

B M2
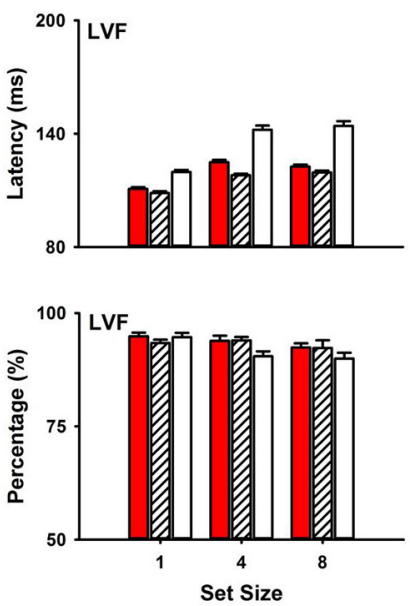

RVF

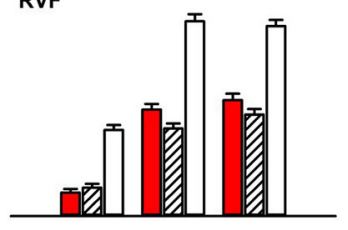

RVF

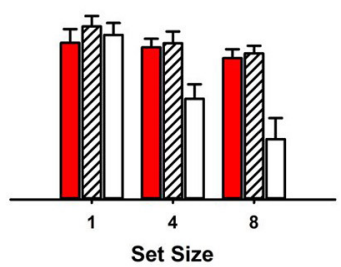

RVF
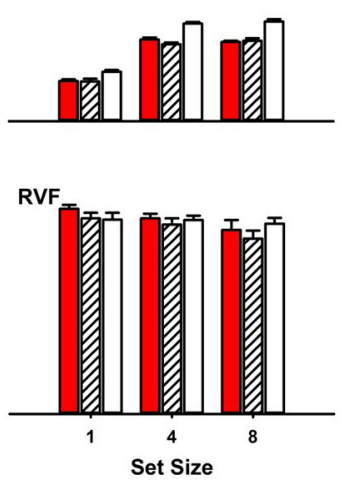

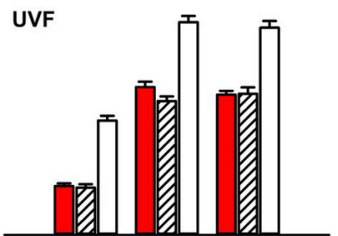

DVF

UVF
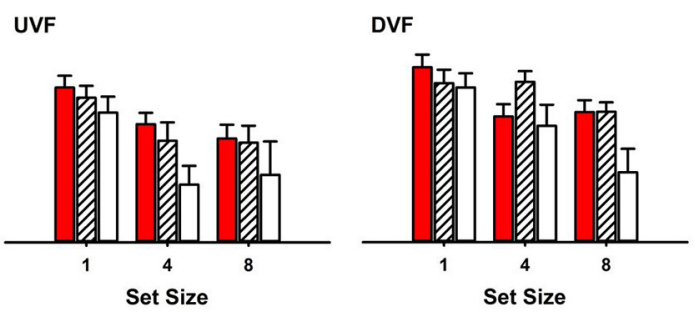

UVF

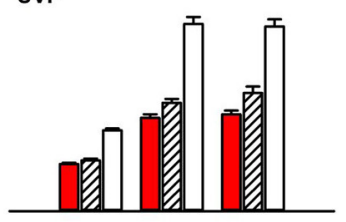

UVF

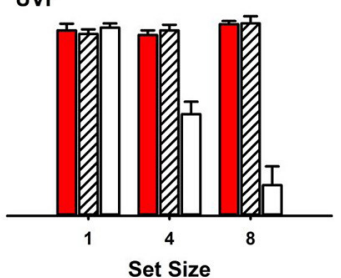

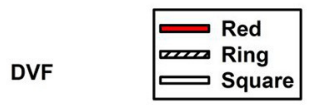
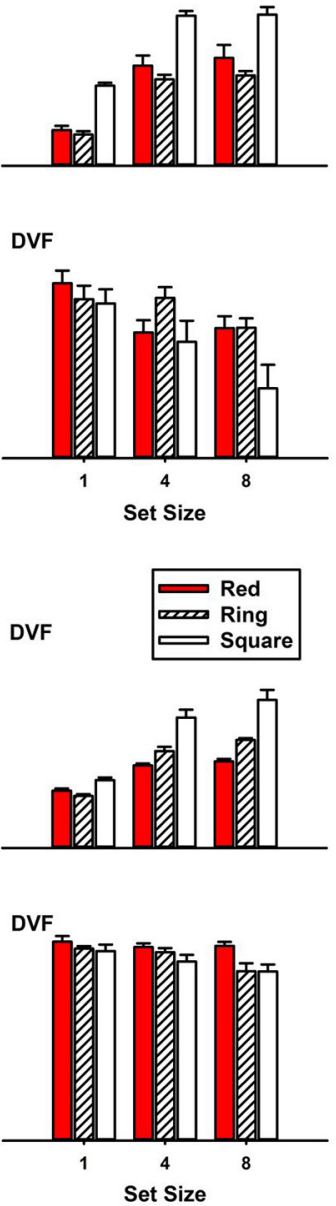

FIGURE 3 | Saccade latencies to targets at eccentricity $4^{\circ}$ in the left, right, upper and down visual fields (LVF, RVF, UVF, and DVF) (A), M1; (B), M2. All percentages of correct trials were large than $80 \%$.

For M1, saccade latencies to the red circle target in the LVF were $141.15 \pm 2.11 \mathrm{~ms}$, which were shorter than that to the yellow (172.24 $\pm 2.19 \mathrm{~ms}, P<0.001)$, green $(173.96 \pm 1.72 \mathrm{~ms}, P<$ $0.001)$ and blue $(227.36 \pm 11.42 \mathrm{~ms}, P<0.001)$ circle targets. Similarly, for M2, saccade latencies to the red circle target (123.42 $\pm 1.48 \mathrm{~ms})$ were shorter than that to the yellow $(148.25 \pm 3.06 \mathrm{~ms}$, $P<0.001)$, green $(147.90 \pm 2.03 \mathrm{~ms}, P<0.001)$ and blue $(163.90$ $\pm 6.20 \mathrm{~ms}, P<0.001$ ) circles (Figure 4). Similar results were obtained for targets with different colors in the RVF.

We further examined the effects of the size of the hole in the ring target on detecting the ring target by comparing saccade latencies to the ring targets with different hole sizes to saccade latencies to the smaller circle targets with similar area differences from the distractors. Linear regressions showed that saccade latencies to the smaller circle targets were dependent on the area differences between the target and the distractors with a slope of $-1.74 \mathrm{~ms}$ per $1 \%$ area difference for M1 (LVF, $P=0.0191$ ) and $-2.51 \mathrm{~ms}$ per $1 \%$ area difference for M2 (LVF, $P=0.0219$ ).
However, saccade latencies to the ring target were independent of the area differences between the target and the distractors because the slopes were not significant from 0 for both monkeys (M1: $-0.57 \mathrm{~ms}$ per $1 \%$ area difference, $P=0.4649$; M2: $-0.37 \mathrm{~ms}$ per $1 \%$ area difference, $P=0.1111$ ) (Figure 5). Similar results were obtained for targets in the RVF of two monkeys. Note that the latency to the smaller circle targets were similar to that to the ring when the area difference from distractor was $50 \%$.

\section{Rapid Detection of a Change in the Global Feature}

Different from the above experiments, in which the target and the distractors were presented simultaneously, in this experiment, the distractor set were presented first followed by replacing one of the distractors with the target. An important difference between the two conditions was that stimulus onset was present in the first condition, but absent in the second condition. Without the stimulus onset, this paradigm allowed us to compare how visual 


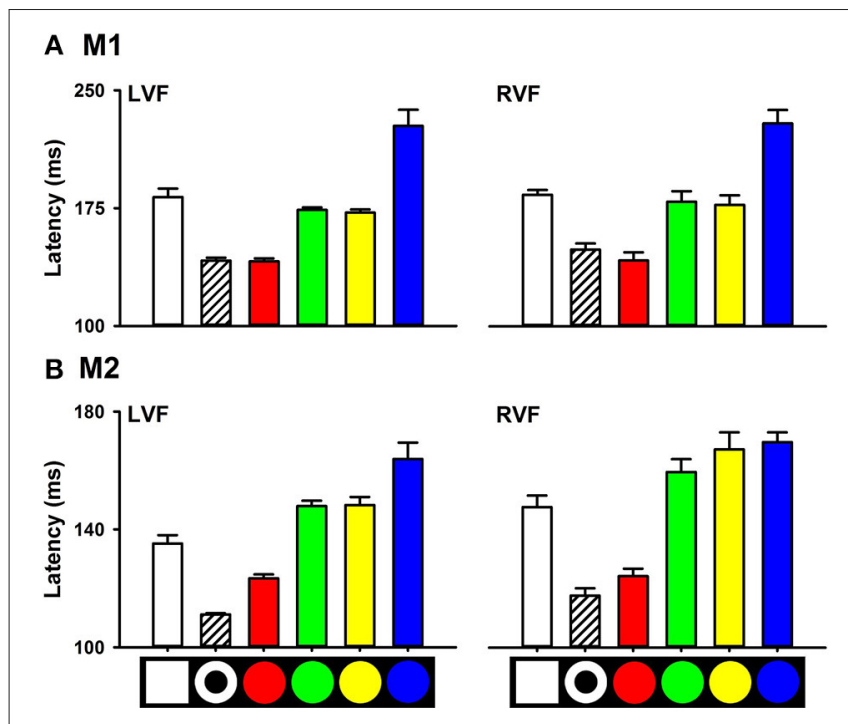

FIGURE 4 | Saccade latencies to targets with different colors (A), M1; (B), M2.

systems detect a change in color, local features, and the global feature. Different from the conditions with target onset, in which detecting the ring was similar to detecting the red circle, in the without target-onset condition, detecting a change in the global feature was faster than detecting a change in color, and local features. For example, in the LVF, saccade latencies of detecting a change in the global feature (M1: $133.18 \pm 2.77 \mathrm{~ms}$; M2: 128.18 $\pm 0.89 \mathrm{~ms}$ ) were shorter than that of detecting a change in color (M1: $170.74 \pm 3.01 \mathrm{~ms}, P<0.001$, Figure 6A, LVF; M2: $146.58 \pm$ $1.05 \mathrm{~ms}, P<0.001$; Figure 6B, LVF) in both monkeys.

\section{Effects of Distinctions in Local and Global Features on Detecting the Distinction in Color}

Distinctions in local and global features exhibited differential effects on detecting the red target from the white distractors. Saccade latencies to the red ring target (M1: $120.85 \pm 1.57 \mathrm{~ms}$; $\mathrm{M} 2: 116.47 \pm 2.38 \mathrm{~ms})$ were significantly shorter than that to the red circle target (M1: $143.24 \pm 3.07 \mathrm{~ms}, P<0.001$; M2: 125.73 $\pm 2.83 \mathrm{~ms}, P<0.001)$. However, saccade latencies to the red square target (M1: $139.12 \pm 1.19 \mathrm{~ms}, P=0.155$; M2: 125.93 $\pm 2.30 \mathrm{~ms}, P=0.848$ ) were not different from that to the red circle target (Figure 7 LVF). Thus, the distinction in the global feature (i.e., ring) facilitates detecting the distinction in color, but the distinction in local features (i.e., square) does not have the facilitating effect. Similar results were obtained for detecting the other colors. For example, the distinction in the global feature facilitated detecting the green ring target in both monkeys [M1: $126.53 \pm 0.72 \mathrm{~ms}$ (Green ring) vs. $166.43 \pm 2.28 \mathrm{~ms}$ (Green disk), $P<0.001 ;$ M2: $117.19 \pm 1.73 \mathrm{~ms}$ (Green ring) vs. $146.73 \pm$ $1.74 \mathrm{~ms}$ (Green disk), $P<0.001$ ], but the distinction in local features slowed down detecting the green square target in one monkey (M1: $170.98 \pm 1.99 \mathrm{~ms}, P=0.026)$ and marginally facilitated in the other monkey (M2: $135.62 \pm 3.05 \mathrm{~ms}, P=0.020)$ (Figure 8). When interpreting rapid detection of red color target,
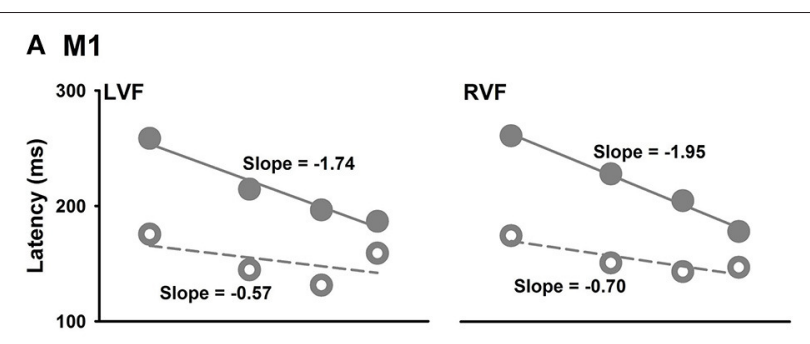

\section{B M2}
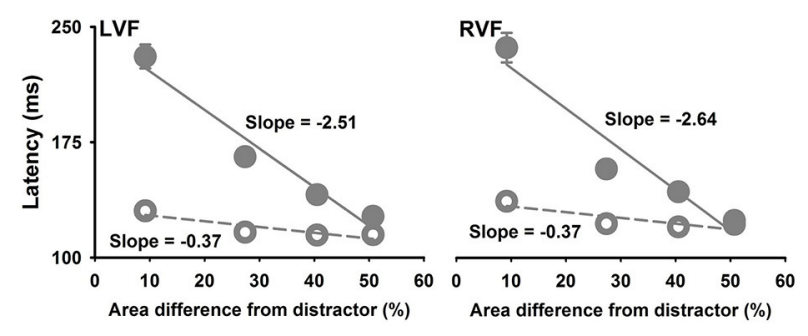

FIGURE 5 | Saccade latencies as a function of the area difference between the target and the distractor. Filled symbols are for the smaller circle target and the open symbols are for the ring target. The unit of slope is ms per $1 \%(\mathbf{A})$, M1; (B), M2.

it is important to rule out luminance as a confounding factor. While the luminance of the red circle target $(43 \mathrm{~cd} / \mathrm{m} 2)$ was lower than that of the white square target $(270 \mathrm{~cd} / \mathrm{m} 2)$, the blue circle target $(76 \mathrm{~cd} / \mathrm{m} 2)$ and the green circle target $(155 \mathrm{~cd} / \mathrm{m} 2)$, detection of the red circle target was faster than that to the other targets. Thus, even though we did not use equiluminant colors, the results indicated that the effects of color could not be accounted for by differences in luminance.

\section{Effects of Distinctions in Local Features on Detecting the Distinction in the Global Feature}

In this experiment, we examined effects of local features of the outside/inside shape of the ring on detecting the ring from the distractors (Figure 9). Interestingly, the two monkeys exhibited different effects. Compared to the standard ring with inside/outside circles, changing to the outside-square ring facilitated its detection for M2, but had no effect for M1. Changing to the inside-square ring slowed its detection for M1, but had no effect for M2. In other words, local features of the outside shape of the ring could facilitate ring's detection, but local features of the inside shape of the ring could slow ring's detection.

\section{DISCUSSION}

In this study, we employed the search detection task to investigate how monkey visual systems process global and local features. Whether a visual object has a hole in it was used as a representative global feature and line segments were used as representative local features. This study was motivated to test a seemly simple prediction of current models of vision. Since 


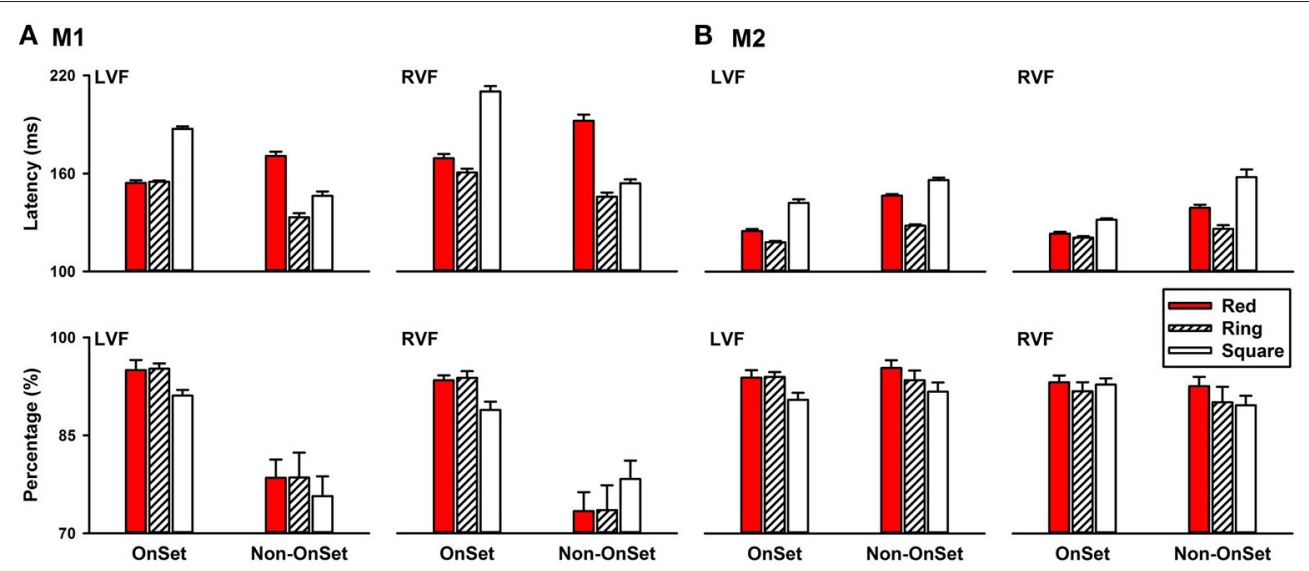

FIGURE 6 | Saccade latencies of detecting a change in color, local features and the global feature in the left, right, upper, and down visual fields (A), M1; (B), M2.

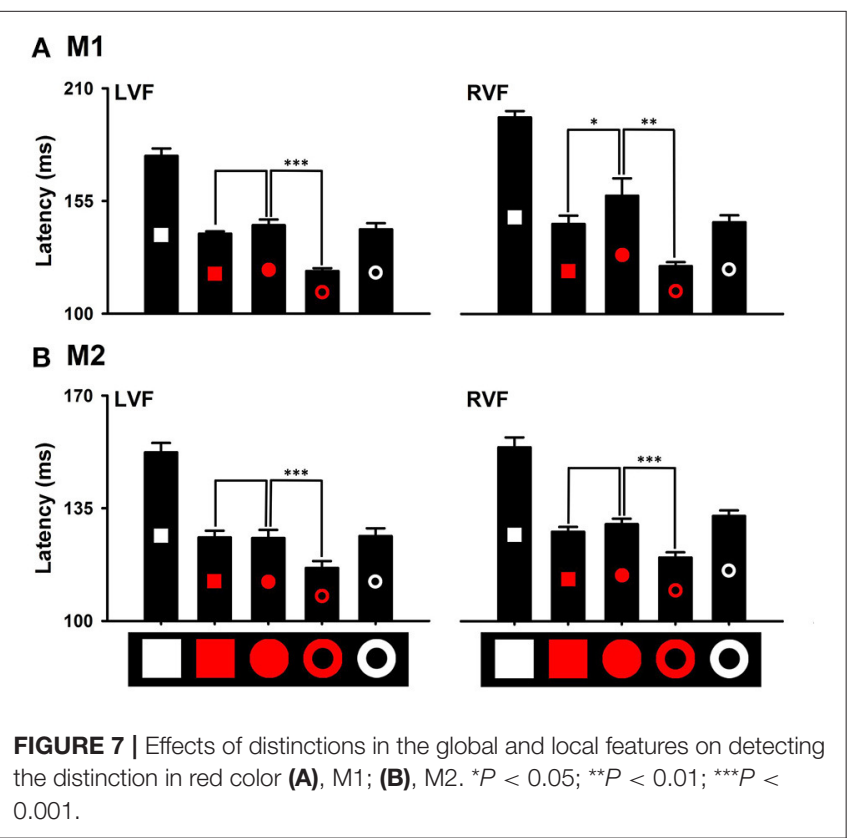

the local features are believed to be extracted in the early visual pathways and the global features are extracted in the later visual pathways by integrating the local features (Figure 10A), it is predicted that detecting distinctions or a change in local features takes less time than detecting distinctions or a change in the global feature. Contrary to this prediction, we found that detecting a distinction in the global feature took less time than detecting distinctions in local features (Figures 3, 6). We further found that detecting a change in the global feature was not only faster than detecting a change in local features, but also faster than detecting a change in color.

\section{Global Feature as a Basic Visual Modality}

Previous studies have demonstrated human visual system early processing of global features characterized as topological

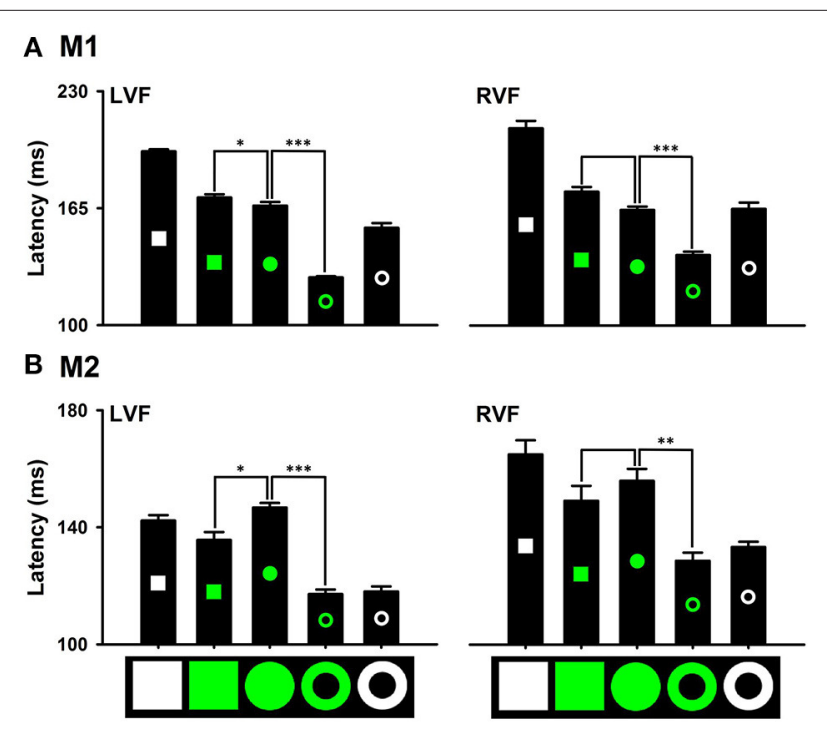

FIGURE 8 | Effects of distinctions in the global and local features on detecting the distinction in green color (A), M1; (B), M2. ${ }^{\star} P<0.05$; ${ }^{\star \star} P<0.01$; ${ }^{\star \star \star} P<$ 0.001 .

invariants, such as whether a visual object has a hole (Chen, 1982, 2005a,b). However, the neural mechanisms underlying processing the global features remain to be elucidated. A critical obstacle is the lack of a non-human primate model of rapid detecting global features for neurophysiological investigations. To the best of our knowledge, this was the first study in behaving monkeys demonstrating rapid processing of a global feature, therefore, filling the important gap, and providing a valuable primate model system for future studies. The behavioral results further put important constraints on models of processing the global and local features and their interactions. For example, is the global feature extracted based on its local features, or as a basic visual modality? The results of this study suggested that the global feature "the object has a hole" is processed as a basic visual modality, likely in parallel with other basic visual modalities, 
such as local features, color, motion, and binocular disparity (Figure 10). This conclusion is based on the following results. First, detecting the global feature took less time than detecting the local features, therefore, it was unlikely based on integrating local features. Second, as shown in Figure 5, saccade latencies of detecting a smaller circle target was dependent on the area difference between the target and the distractor, but detecting the ring target was not affected by its area difference with the distractor. Therefore, rapid detection of the ring was not due to its area difference to the distractor, but due to the distinction in the global feature, i.e., the ring had a hole but the circle distractor did not. Third, detecting a change in the global feature was not

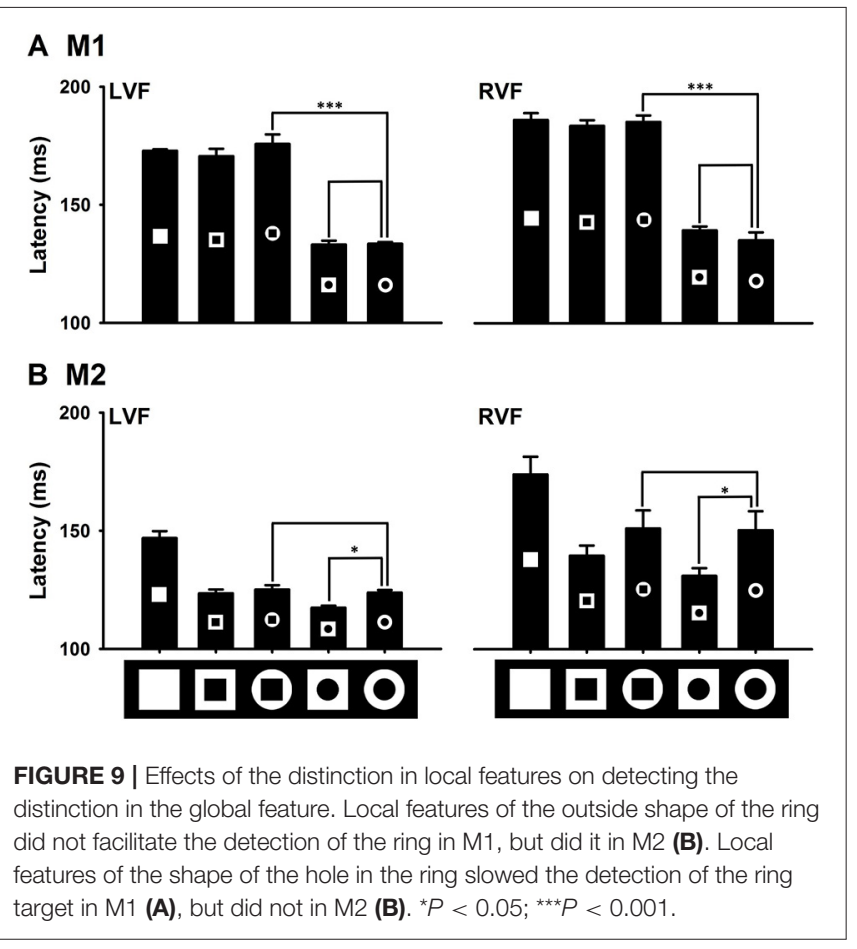

only faster than detecting a change in local features, but also faster than detecting a change in color. Taken together, these behavioral results suggest that processing the global feature, i.e., number of holes in a visual object, is not based on processing local features, but in parallel or even prior to it.

\section{The Superior Colliculus (SC) as a Potential Site for Rapid Processing of the Global Feature}

It has been well-established that the local features are first processed by the primary visual cortex, which receives retinal inputs via the lateral geniculate body pathways, and then integrated by the visual areas along the dorsal pathways, such as V2, V4, and inferior temporal cortex, which have neurons tuning to more complicated visual features, e.g., face and hand (Van Essen and Maunsell, 1983; Livingston and Hubel, 1988). However, little is known about where in the visual systems the global feature is processed. In fact, there is no study that directly examines the neural substrates that process the global features, such as whether an object has a hole. We suggest that the global feature is unlikely to be processed by the primary visual cortex because the global feature is directly extracted from the whole object, rather than by integrating its simple components, such as line segments. The global feature may be at least preliminarily processed by the superior colliculus (SC), which receives substantial direct retinal inputs and project to the cortical visual areas via the pulvinar nucleus of the thalamus (Pessoa and Adolphs, 2010). In the past several decades, visual responses of SC neurons have been extensively studied, but little is known on what the specific visual features are extracted by the SC neurons.

There are several lines of evidence suggesting a role of the SC in processing the global features. First, the SC neurons respond to the onset of visual targets without specific requirements for its local features. In other words, the SC neurons encode whether there is a new object in the scene, which is a global feature. Second, as shown by the seminal studies of Basso and

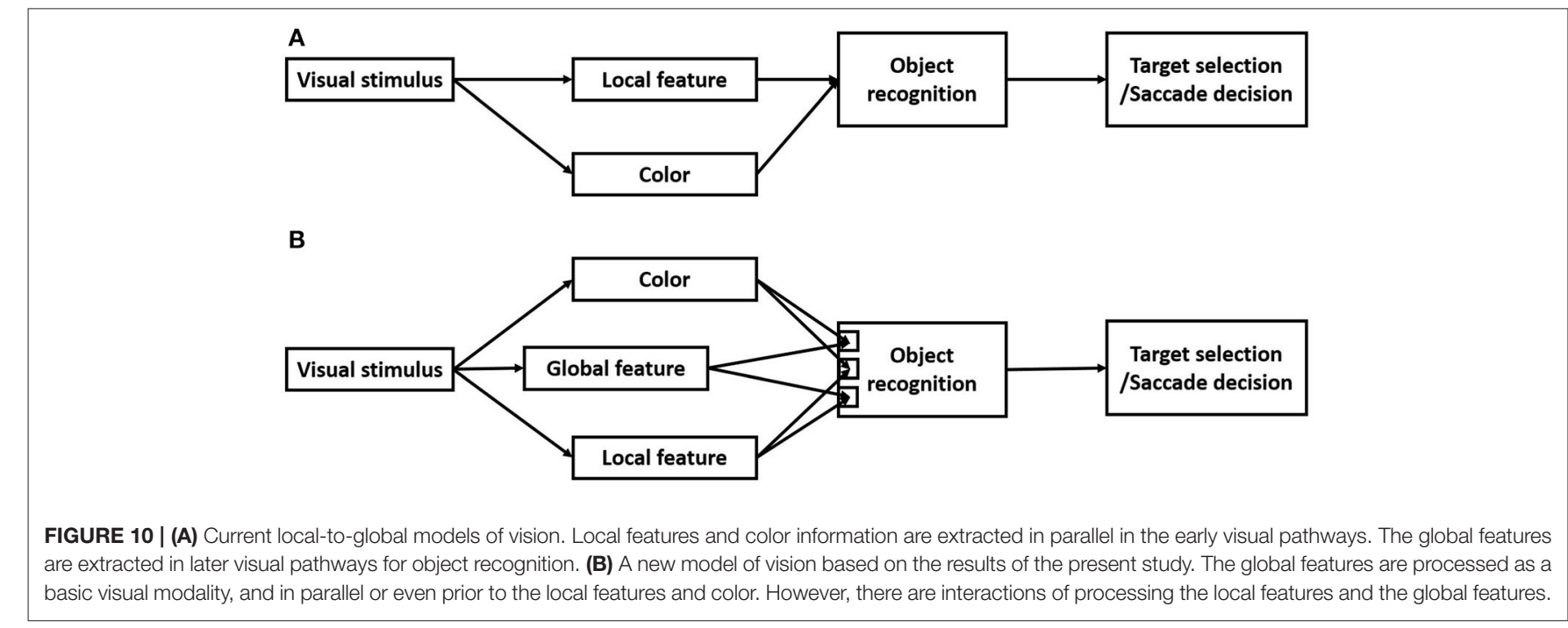


Wurtz (1997, 1998), the visual responses of the SC neurons were modulated by the number of objects in the visual display or target uncertainty. Although the authors showed that the SC neurons did encode target uncertainty defined by factors other than visual configuration information, it is important to note that the SC neurons did encode the number of potential targets in each trial. Again, similar to target onset, which indicates the appearance of a new object in the scene, number of objects in the scene is also a global feature independent of local features. Since the latency of the target uncertainty effect was very short, nearly at the onset of visual responses of SC neurons, it is possible that number of visual objects in the display is processed within the SC circuits, without the need of cortical feedbacks. Third, neurons in the SC and V1 exhibit strikingly different responses to retinal image motion caused by the movement of an object in the world or of the eye itself across the visual scene. Robinson and Wurtz (1976) showed that most of the SC neurons differentiated external stimulus movement from image movement resulting from a monkey's own eye movement. Thus, the SC neurons would provide an uncontaminated visual signal to their target areas to facilitate decision making and sensorimotor transformation. In contrast to SC neurons, however, Wurtz (1969) showed that the V1 neurons did not discriminate between the stimulus movement and eye movement conditions. Fourth, the behavioral results showed that a distinction in local features did not speed up detecting a distinction in color, but a distinction in the global feature did. This finding is consistent with the study of White et al. (2009), which showed that the SC neurons effectively processed color information.

In summary, this study achieved two goals. First, we established a non-human primate model of rapid detection of a global feature of a visual object, i.e., whether it has a hole. This model lay the foundation for studying the neural mechanisms underlying differential processing of global and local features for visual object recognition. Second, the results put important constraints on future models of vision, which

\section{REFERENCES}

Ahissar, M., and Hochstein, S. (2004). The reverse hierarchy theory of visual perceptual learning. Trends Cogn. Sci. 8, 457-464. doi: 10.1016/j.tics.2004. 08.011

Basso, M. A., and Wurtz, R. H. (1997). Modulation of neuronal activity by target uncertainty. Nature 389, 66-69. doi: 10.1038/37975

Basso, M. A., and Wurtz, R. H. (1998). Modulation of neuronal activity in superior colliculus by changes in target probability. J. Neurosci. 18, 7519-7534.

Bichot, N. P., and Schall, J. D. (1999). Saccade target selection in macaque during feature and conjunction visual search. Vis. Neurosci. 16, 81-89. doi: $10.1017 /$ S0952523899161042

Bona, S., Cattaneo, Z., and Silvanto, J. (2016). Investigating the causal role of rOFA in holistic detection of mooney faces and objects: an fMRI-guided TMS study. Brain Stimul. 9, 594-600. doi: 10.1016/j.brs.2016.04.003

Bronstein, A. M., and Kennard, C. (1987). Predictive saccades are different from triggered saccades. Vision Res. 27, 517-520. doi: 10.1016/0042-6989 (87) $90037-X$

Bruce, C. J. (1982). Face recognition by monkeys: absence of an inversion effect. Neuropsychologia 20, 515-521. doi: 10.1016/0028-3932(82)90025-2 should treat the global feature as a basic visual modality like local features. It is worth noting that detecting local features and the global feature exhibited non-linear interactions. In particular, the local features of the hole of the ring slowed down detection of the hole, indicating possible interactions between the primary visual areas and the SC. Thus, new models should allow interactions between the modules of detecting global features and the modules of detecting local features in order to generate the observed behaviors. Future studies will further investigate a role of the subcortical pathways in processing global features in behaving monkeys. The new information will not only fill the current knowledge gap, but also provide constraints on new neural models of visual information processing.

\section{AUTHOR CONTRIBUTIONS}

Study conception and design: WZ, YZ, JH, YaY, KZ. Acquisition of data: JH, YaY, XZ, QZ, HZ, YiY, CZ. Analysis and interpretation of data: WZ, YZ, JH, YaY, KZ. Drafting of manuscript: WZ, YZ, JH, YaY and KZ.

\section{FUNDING}

Supported by NIH grants to WZ (R01DC008585, R21EY025550, and R01DC012060), the National Natural Science Foundation of China to YZ (NSFC 31230032), Key Research Program of Frontier Sciences, Chinese Academy of Sciences to YaY (QYZDBSSW-SMC032), National Nature Science Foundation of China grant (31671133) to KZ and Guangdong Pearl River Talents Plan Innovative and Entrepreneurial Team grant (2016ZT06S220) to $\mathrm{KZ}$.

\section{ACKNOWLEDGMENTS}

We thank Jiachun Cai for writing the data acquisition program and Jerome Allison for technical assistance.

Bruce, C. J., Desimone, R., and Gross, C. G. (1981). Visual properties of neurons in a polysensory area in superior temporal sulcus of the macaque. J. Neurophysiol. 46, 369-384.

Chen, L. (1982). Topological structure in visual perception. Science 218, 699-700. doi: $10.1126 /$ science.7134969

Chen, L. (1985). Topological structure in the perception of apparent motion. Perception 14, 197-208. doi: 10.1068/p140197

Chen, L. (1990). Holes and wholes: a reply to Rubin and Kanwisher. Percept. Psychophys. 47, 47-53. doi: 10.3758/BF03208163

Chen, L. (2005a). Reply author's response where to begin. Vis. Cogn. 12, 691-701. doi: 10.1007/978-3-319-44832-9_7

Chen, L. (2005b). The topological approach to perceptual organization. Vis. Cogn. 12, 553-637. doi: 10.1080/13506280444000364

Chen, L., and Zhou, W. (1997). Holes in illusory conjunction. Psychon. Bull. Rev. 4, 507-511. doi: 10.3758/BF03214340

Chen, L., Zhang, S., and Srinivasan, M. V. (2003). Global perception in small brains: Topological pattern recognition in honey bees. Proc. Natl. Acad. Sci. U.S.A. 100, 6884-6889. doi: 10.1073/pnas.0732090100

Desimone, R. (1991). Face-selective cells in the temporal cortex of monkeys. $J$. Cogn. Neurosci. 3, 1-8. doi: 10.1162/jocn.1991.3.1.1 
Fischer, B., and Weber, H. (1993). Express saccades and visual attention. Behav. Brain Sci. 16, 553-610. doi: 10.1017/S0140525X00031575

Fischer, B., Weber, H., Biscaldi, M., Aiple, F., Otto, P., and Stuhr, V. (1993). Separate populations of visually guided saccades in humans: reaction times and amplitudes. Exp. Brain Res. 92, 528-541. doi: 10.1007/BF002 29043

Gross, C. G., Rocha-Miranda, C. E., and Bender, D. B. (1972). Visual properties of neurons in inferotemporal cortex of the macaque. J. Neurophysiol. 35, 96-111.

Hubel, D. H. (1982). Exploration of the primary visual cortex. Nature 299, 515-524. doi: $10.1038 / 299515 \mathrm{a} 0$

Hubel, D. H. (1988). Eye, Brain, and Vision. New York, NY: Scientific American Library.

Hubel, D. H., and Wiesel, T. N. (1962). Receptive fields, binocular interaction and functional architecture in the cat's visual cortex. J. Physiol. 160, 106-154. doi: 10.1113/jphysiol.1962.sp006837

Hubel, D. H., and Wiesel, T. N. (1963). Shape and arrangement of columns in cat's striate cortex. J. Physiol. 165, 559-568. doi: 10.1113/jphysiol.1963.sp007079

Hubel, D. H., and Wiesel, T. N. (1977). Ferrier lecture. Functional architecture of macaque monkey visual cortex. Proc. B. Soc. Lond. B. 198, 1-59. doi: $10.1098 /$ rspb.1977.0085

Judge, S. J., Richmond, B. J., and Chu, F. C. (1980). Implantation of magnetic search coils for measurements of eye position: an improved method. Vision Res. 20, 535-538. doi: 10.1016/0042-6989(80)90128-5

Kalesnykas, R. P., and Hallet, P. E. (1987). The differentiation of visually guided and anticipatory saccades in gap and overlap paradigms. Exp. Brain Res. 68, 115-121. doi: 10.1007/BF00255238

Komatasu, H., and Ideura, Y. (1993). Relationships between color, shape, and pattern selectivities of neurons in the inferior temporal cortex of the monkey. J. Neurophysiol. 70, 677-694.

Livingston, M., and Hubel, D. (1988). Segregation of form, color, movement, and depth: anatomy, physiology, and perception. Science 240, 740-749. doi: $10.1126 /$ science. 3283936

Marr, D. (1982). Vision: A Computational Investigation into the Human Representation and Processing of Visual Information. New York, NY: Freeman.

Navon, D. (1977). Forest before trees: The precedence of global features in visual perception. Cogn. Psychol. 9, 353-383. doi: 10.1016/0010-0285(77)90012-3

Pare, M., and Munoz, D. P. (1996). Saccadic reaction time in the monkey: advanced preparation of oculomotor program is primarily responsible for express saccade occurrence. J. Neurophysiol. 76, 3666-3681.

Pessoa, L., and Adolphs, R. (2010). Emotion processing and the amygdala: from a low road to many roads of evaluating biological significance. Nat. Rev. Neurosci. 11, 773-783. doi: $10.1038 / \mathrm{nrn} 2920$

Robinson, D. A. (1963). A method of measuring eye movement using a scleral search coil in a magnetic field. IEEE Trans. Biomed. Eng. 10, 137-145.

Robinson, D. E., and Wurtz, R. H. (1976). Use of an extraretinal signal by monkey superior colliculus neurons to distinguish real from self-induced stimulus movement. J. Neurophysiol. 39, 852-870.

Schwartz, E. L., Desimone, R., Albright, T. D., and Gross, C. G. (1983). Shape recognition and inferior temporal neurons. Proc. Natl. Acad. Sci. U.S.A. 80, 5776-5778. doi: 10.1073/pnas.80.18.5776

Shen, Z., Zhang, W., and Chen, Y. (2002). The hole precedence in face but not figure discrimination and its neuronal correlates. Vision Res. 42, 873-882. doi: 10.1016/S0042-6989(01)00316-9

Smith, A. C., and Van Gisbergen, J. A. M. (1989). A short-latency transition in saccade dynamics during square-wave tracking and its significance for the differentiation of visually-guided and predictive saccades. Exp. Brain Res. 76, 64-74. doi: 10.1007/BF00253624
Tootell, R. B., Hadjikhani, N. K., Vanduffel, W., Liu, A. K., Mendola, J. D., Sereno, M. I., et al. (1998). Functional analysis of primary visual cortex (V1) in humans. Proc. Natl. Acad. Sci. U.S.A. 95, 811-817. doi: 10.1073/pnas.95.3.811

Treisman, A. M., and Gelade, G. (1980). A feature-integration theory of attention. Cogn. Psychol. 12, 97-136. doi: 10.1016/0010-0285(80)90005-5

Van Essen, D., and Maunsell, J. H. R. (1983). Hierarchical organization and functional streams in the visual cortex. Trends Neurosci. 6, 370-375. doi: 10.1016/0166-2236(83)90167-4

Van Hooser, S. D., Roy, A., Rhodes, H. J., Culp, J. H., and Fitzpatrick, D. (2013). Transformation of receptive field properties from lateral geniculate nucleus to superficial V1 in the tree shrew. J. Neurosci. 33, 11494-11505. doi: 10.1523/JNEUROSCI.1464-13.2013

Wang, B., Zhou, T., Zhuo, Y., and Chen, L. (2007). Global topological dominance in the left hemisphere. Proc. Natl. Acad. Sci. U.S.A. 104, 21014-21019. doi: 10.1073/pnas.0709664104

Weisstein, N., and Harris, C. S. (1974). Visual detection of line segments: an object-superiority effect. Science 186:752. doi: 10.1126/science.186.4165.752

Wenban-Smith, M. G., and Findlay, J. M. (1991). Express saccades: is there a separate population in humans?. Exp. Brain Res. 87, 218-222. doi: $10.1007 / \mathrm{BF} 00228523$

White, B. J., Boehnke, S. E., Marino, R. A., Itti, L., and Munoz, D. P. (2009). Colorrelated signals in the primate superior colliculus. J. Neurosci. 29, 12159-12166. doi: 10.1523/JNEUROSCI.1986-09.2009

Wurtz, R. H. (1969). Comparison of effects of eye movements and stimulus movements on striate cortex neurons of the monkey. J. Neurophysiol. 32, 987-994.

Yacoub, E., Harel, N., and Ugurbil, K. (2008). High-field fMRI unveils orientation columns in humans. Proc. Natl. Acad. Sci. U.S.A. 105, 10607-10612. doi: 10.1073/pnas.0804110105

Yeh, C., Xing, D., and Shapley, R. M. (2009). Black responses dominate macaque primary visual cortex V1. J. Neurosci. 29, 11753-11760. doi: 10.1523/JNEUROSCI.1991-09.2009

Zhou, K., Luo, H., Zhou, T., Zhuo, Y., and Chen, L. (2010). Topological change disturbs object continuity in attentive tracking. Proc. Natl. Acad. Sci. U.S.A. 107, 21920-21924. doi: 10.1073/pnas.1010919108

Zhou, W., and King, W. M. (1998). Premotor commands encode monocular eye movements. Nature 393, 692-695. doi: 10.1038/31489

Zhou, W., and King, W. M. (2002). Attentional sensitivity and asymmetries of vertical saccade generation in monkey. Vision Res. 42, 771-779. doi: 10.1016/S0042-6989(01)00319-4

Zhuo, Y., Zhou, T., Rao, H., Wang, J., Meng, M., Chen, M., et al. (2003). Contributions of the visual ventral pathway to long-range apparent motion. Science 299, 417-420. doi: 10.1126/science.1077091

Zoccolan, D. (2015). Invariant visual object recognition and shape processing in rats. Behav. Brain Res. 285, 10-33. doi: 10.1016/j.bbr.2014.12.053

Conflict of Interest Statement: The authors declare that the research was conducted in the absence of any commercial or financial relationships that could be construed as a potential conflict of interest.

Copyright () 2017 Huang, Yang, Zhou, Zhao, Zhou, Zhu, Yang, Zhang, Zhou and Zhou. This is an open-access article distributed under the terms of the Creative Commons Attribution License (CC BY). The use, distribution or reproduction in other forums is permitted, provided the original author(s) or licensor are credited and that the original publication in this journal is cited, in accordance with accepted academic practice. No use, distribution or reproduction is permitted which does not comply with these terms. 